magnetic lenses with gradients acting on the dipole moments of the molecules or atoms. Finally, it is not necessary to be limited to thermally induced evaporation (self-luminous objects); atoms can be scattered or condensed and evaporated from the surface of various samples. Eventually, it should be possible to achieve quite high magnification in several stages, by always taking advantage of the power of low-temperature vacuum techniques for obtaining extremely small backgrounds of undesired atoms and molecules. Interesting biological applications could then arise.

We thank R. M. Koolish and F. J. O'Brien for assistance with apparatus, E. H. Jacobsen for discussions, B. J. Wuensch for providing the feldspar sample and advice and H. F. Dylla for aid in the $\mathrm{K}_{2} \mathrm{O}$ vapour pressure measurements. This work was supported by the Joint Services Electronics Program.

JoHN G. KING

William R. Bigas

Research Laboratory of Electronics and

Department of Physics,

Massachusetts Institute of Technology,

Cambridge, Massachusetts 02139.

Received September 13, 1968.

${ }^{1}$ Ramsey, N. F., Molecular Beams (Oxford Univ. Press, London, 1956). King, J. G., and Zacharias, J. R., Advances in Electronics and Electron Physics, 8 (A Academic Press, New Y̧ork, 1956).

${ }^{2}$ Johnston, jun., W. D., and King, J. G., Phys. Rev. Lett., 16, 1191 (1966); Rev. Sci. Instrum, 37, $475(1066)$

"Woods, R. D., and Fenn, J. B., Rev. Sci. Instrum., 37, 917 (1966).

\section{Electron Microscopy of Sodium Montmorillonite}

ThE two-dimensional structure of montmorillonite has been illustrated by electron micrographs of the face view

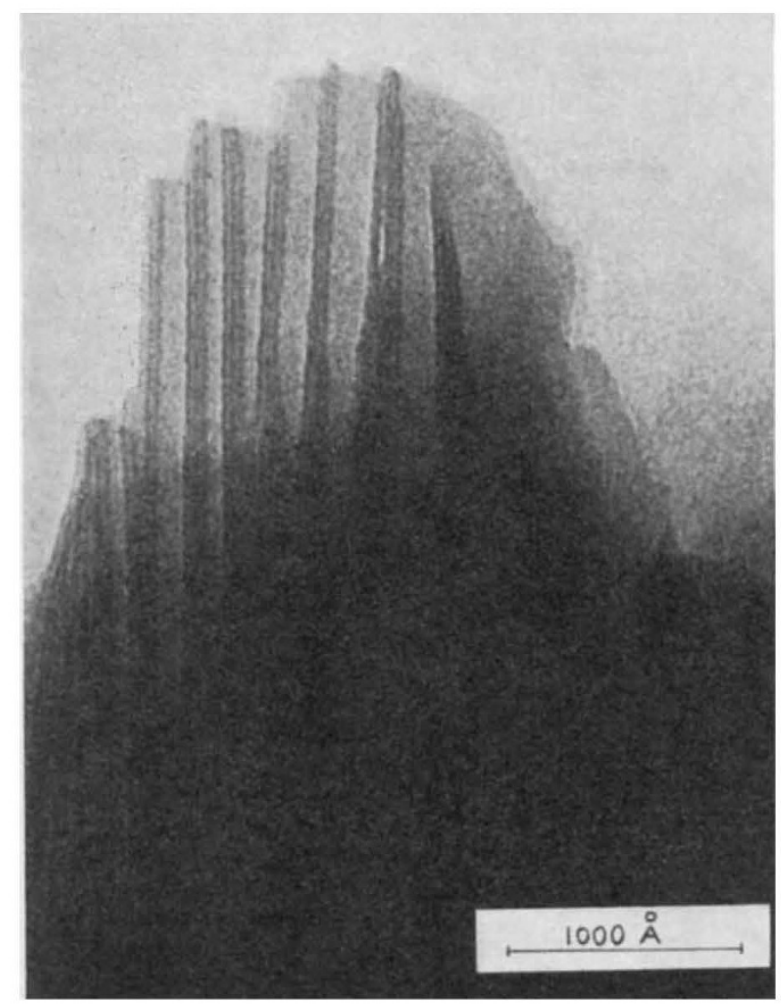

Fig. 1. of the plates ${ }^{1-3}$. We report what we believe to be the first micrograph to be published of the edge view of the unit layers of montmorillonite. In Fig. 1 the plates appear to be supported at an angle to the supporting film; the dark parallel lines, which are $10 \AA \pm 2 \AA$, are the unit layers of montmorillonite. This is in agreement with $\mathrm{X}$-ray data which give the unit layer thickness as approximately $10 \AA$. It is interesting to note that the lines appear in groups of three or four.

The sample was prepared by dispersing dry crude montmorillonite (Wyoming Bentonite) in water, containing $1 \mathrm{ml}$. of 30 per cent hydrogen peroxide/100 g of clay (to oxidize any organic matter) and centrifuging to remove gritty impurities such as silica. The sample was converted to the sodium form of montmorillonite by redispersing the clay in molar sodium chloride and allowing this to stand for several days. The excess salt was romoved by dialysis with water. On centrifuging the dispersion at $38,000 \mathrm{~g}$ for $\mathrm{l} \mathrm{h}$, the montmorillonite separated out as a gel, which appeared transparent yellow at the top of the centrifuge tube with gradations to an opaque yellow colour at the bottom, resulting from a distribution of plate size. The yellow colour is attributed to traces of iron or chromium present in octahedral lattice sites.

Some of the opaque fraction, consisting of the larger plates (approximately 1 micron in size), was dispersed in water. A sample of this was spotted on to plastic supporting films and was studied at an accelerating potential of $50 \mathrm{kV}$ in a Hitachi $H U 11 \mathrm{~B}$ electron microscope. The edges of plates were observed in small areas on several microscope grids.

L. M. BARCLAY *

D. W. Thompson

School of Chemistry,

University of Bristol.

Received Fcbruary 24, 1969.

*Present address: BP Chemicals (UK) Ltd, 13P Plastics Department, Barry, Glamorganshire.

${ }^{1}$ van Olphen, H., in An Introduction to Clay Colloid Chemistry (Interscience, 1963).

${ }^{2}$ Corbet, H. C., and Wolffes, J., Proc. Stockholm Conf. on Elect. Microse., 334

(1956).
${ }^{3}$ Bates, T. F., and Comer, J. J., Proc. of the Third Nat. Conf. on Clay and Clay Min., Publ. 395, 1 (1955).

\section{Visual Observation of Surface Vibration Nodal Patterns}

SEVERAL methods for studying surface vibration modes using holography have recently been developed ${ }^{1-3}$. These effectively contour the surface with interference fringes, which delineate points of equal amplitude of vibration. By using stroboscopic methods in conjunction with holography, the relative phase of different points can also be measured ${ }^{3-5}$. We describe here a somewhat simpler method of vibration analysis, which does not involve the photographic process, and does not need the stability against long term drift effects that is essential to the recording of a hologram. It is, however, capable of determining only the nodal areas. It is essentially an observational method, depending on the appearance of the speckle pattern that is seen when the surface of an object is illuminated with coherent light.

The formation of laser speckle is a result of the interaction of individual coherent waves, scattered by the surface into the direction of observation. For any elementary area of surface resolved by the viewing system, those waves will sum together vectorially to give a finite value of intensity, which will vary from one elementary area to the next. The statistics governing the distribution of light in the speckle pattern have been treated by Rayleigh ${ }^{6}$ and Goldfischer ${ }^{7}$. Here it is important to note 